\title{
Role of F18-FIDG-PET/CT' in restaging patients affected by renal carcinoma
}

\author{
Francesco Bertagna', Federica Motta ${ }^{1}$, Mattia Bertoli ${ }^{1}$, \\ Giovanni Bosio', Simona Fisogni², Regina Tardanico³, \\ Vittorio Ferrari ${ }^{4}$, Alessandro Antonelli ${ }^{5}$, Claudio Simeone ${ }^{5}$, \\ Sergio Cosciani Cunico ${ }^{5}$, Raffaele Giubbini ${ }^{1}$ \\ ${ }^{1}$ Nuclear Medicine, University of Brescia and Spedali Civili \\ di Brescia, Brescia, Italy \\ ${ }_{21} 1^{\text {st }}$ Division of Pathological Anatomy, University of Brescia and \\ Spedali Civili di Brescia, Brescia, Italy \\ ${ }^{3} 2^{\text {nd }}$ Division of Pathological Anatomy, University of Brescia and \\ Spedali Civili di Brescia, Brescia, Italy \\ ${ }^{4}$ Oncology Department, Spedali Civili di Brescia, Brescia, Italy \\ ${ }^{5}$ Chair of Urology, University of Brescia and Spedali Civili di \\ Brescia, Brescia, Italy
}

[Received 9 III 2012; Accepted 28 XI 2012]

\begin{abstract}
BACKGROUND: Renal cancers account for around 3\% of all cancers and the most common type of $(90 \%)$ is renal cell carcinoma Five-year survival rate in renal cancer patients is $68.4 \%$. AIM: The aim of our study was to establish the role of F18-FDG-PET/CT in restaging patients with renal carcinoma who underwent partial or radical nephrectomy. Secondary aim of the study was to identify histological characteristics of the primary tumour that may be responsible for the metabolic behaviour of neoplastic lesions.
\end{abstract}

MATERIALS AND METHODS: We retrospectively evaluated 68 patients with renal carcinoma in whom F18-FDG-PET/CT was performed.

RESULTS: Sensitivity, specificity, positive predictive value, negative predictive value and accuracy of F18-FDG PET/CT were $82 \%, 100 \%, 100 \%, 66.7 \%$ and $86.8 \%$, respectively.

CONCLUSIONS: The results of our study suggest that F18-FDG $\mathrm{PET} / \mathrm{CT}$ is characterised by high specificity and positive predictive value and can be useful in restaging patients affected by

Correspondence to: Francesco Bertagna, MD

Chair of Nuclear Medicine, University of Brescia and Spedali Civili di Brescia P.le Spedali Civili, 1

25123 Brescia, Italy

Tel: + 39303995468

Fax: + 39303995420

e-mail: francesco.bertagna@spedalicivili.brescia.it,

francesco.bertagna@med.unibs.it renal carcinoma. However, due to low negative predictive value, this method cannot be recommended for definitely ruling out suspected disease relapse.

KEY words: PET/CT, F18-FDG, renal cell carcinoma

Nuclear Med Rev 2013; 16, 1: 3-8

\section{Background}

Renal cancers account for around $3 \%$ of all cancers [1] and the most common type (90\%) is renal cell carcinoma (RC) [2]. Five-year survival rate in renal cancer patients is $68.4 \%$ all over the globe [3]. $\mathrm{RC}$ has been shown to be resistant to radiation therapy and chemotherapy [4], therefore radical or partial nephrectomy remains the mainstay of treatment for the localized disease [5]. Although patients with RC localized in the kidney are treated with surgery and have a favourable prognosis [5], 20-40\% will later develop metastases and the 5-year survival decreases to below 10\% [6]. Furthermore, $20-30 \%$ of the patients already have metastatic disease at the time of diagnosis [7]. The most common sites for the recurrences are the lung and regional lymph nodes followed by bone, liver and brain [8]. In the past, treatment of metastatic $\mathrm{RC}$ (MRC) was limited to interferon-a, interleukin-2, cytoreductive surgery, and palliative radiation therapy, but recently therapy with sunitinib, sorafenib $[9,10]$, multiple tyrosine kinase inhibitors (multiple TKIs) targeting vascular endothelial growth factor receptor (VEGFR), and everolimus [11] has been proposed as new options. Ultrasonography (US), contrast enhanced computed tomography (CECT) and magnetic resonance imaging (MRI) are commonly used imaging modalities for the detection of $\mathrm{RC}$ recurrences, providing morphological details. Prognosis in RC depends on tumour extent, presence of metastasis and other prognostic factors, like age, general state of health, and co-morbidities. Unfortunately there is no specific laboratory parameter useful and reliable as a tumour marker for RC $[12,13]$. The primary aim of our study was to establish the role of F18-FDG PET/CT in restaging patients affected by $\mathrm{RC}$ who underwent partial or radical nephrectomy. The reference standard was considered histology of the lesions, when available, or clinical evaluation plus all radiologic studies performed (CECT, MRI, US). Secondary aim of the study was to identify histological characteristics of the primary tumour that may be responsible for the metabolic behaviour of neoplastic lesions and, consequently, for F18-FDG-PET/CT results. 


\section{Materials and methods}

From August 2005 to July 2011, about 9600 patients were evaluated and $14800 \mathrm{PET} / \mathrm{CT}$ studies were performed in our department of Nuclear Medicine. Among this large group of patients, only in 68 (49 male [72\%] and 19 female [28\%]; median age: $68 \pm 4$ years) had a diagnosis of renal carcinoma (confirmed after surgical intervention). We retrospectively evaluated these patients. In particular, 62 patients (91\%) underwent total nephrectomy and 6 (9\%) partial nephrectomy. Data regarding histological subtype of renal carcinoma was available in 52 patients (76.5\%); a clear cell renal cell carcinoma was diagnosed in 44 patients $(64.7 \%)$, a chromophobe renal cell carcinoma in $4(5.8 \%)$, a papillary cell carcinoma in $3(4.5 \%)$, and a collecting ducts (Bellini) carcinoma in 1 (1.5\%); for the remaining 16 patients (23.5\%) the histological subtype data was not available. According to Fuhrman grading score (available for all 44 patients with clear cell carcinoma) of primary tumour [14], no patient was classified as G1, 12 patients (27\%) were classified as G2, 15 patients (34\%) as G3 and 17 patients (39\%) as G4.

All patients underwent F18-FDG PET/CT examination in order to restage the disease due to clinical suspicion of disease relapse or during routine follow-up. The reference standard to assess PET diagnostic accuracy was considered histology of secondary lesions (available in 10 patients) or the sum of clinical and all radiological data available (CECT, MRI, US). Moreover, 11 patients after the first F18-FDG PET/CT examination underwent a second study to assess response to systemic therapy with sunitinib or sorafenib or to local radiation therapy.

F18-FDG PET/CT was performed after at least 6 hours fasting and with the glucose level lower than $150 \mathrm{mg} / \mathrm{dl}$. FDG dose of $5.5 \mathrm{MBq} / \mathrm{Kg}$ was administered intravenously and a $2 \mathrm{D}$ mode ordered-subset-expectation-maximization (OS-EM) imaging (with septa) was acquired 60 minutes after injection from the skull basis to the mid-thigh on a Discovery ST PET/CT tomograph (General Electric Company - GE ${ }^{\circledR}$ - Milwaukee, WI, USA) with standard CT parameters $(80 \mathrm{~mA}, 120 \mathrm{Kv}$ without contrast; 4 minutes per bed-PET-step of $15 \mathrm{~cm}$ ). The reconstruction was performed in a $128 \times 128$ matrix and $60 \mathrm{~cm}$ field of view. The patients were instructed to void before imaging. The PET images were analysed visually and semi-quantitatively by measuring the maximum standardized uptake value (SUVmax). SUV was expressed as SUVbody weight (SUVbw - g/ml) and automatically calculated by the software (Volumetrix for PET/CT; Xeleris ${ }^{\text {TM }}$ Functional imaging workstation; GE) on the basis of following parameters: weight of the patient expressed in kilograms; height expressed in centimetres; tracer volume expressed in $\mathrm{ml}$; radioactivity at injection time expressed in MBq; post injection activity in the vial expressed in $\mathrm{MBq}$; injection time; starting time acquisition; decay half-time of the radioisotope (standard 109.8 minutes for F18-FDG). A written consensus was obtained from all patients before every study.

Images readout was performed on a Xeleris ${ }^{\text {TM }}$ Functional imaging workstation $\left(\mathrm{GE}^{\circledR}\right)$ and the reader had knowledge of the clinical history. Every focal tracer uptake deviation from physiological distribution was considered as suggestive of diseases while faint bilateral tracer uptake in inguinal, axillary and pulmonary hilar lymph-nodes was considered as being inflammatory. No specific SUV-value cut-off was adopted to discriminate benign from malignant lesions. Instead, the diagnostic criterion was the deviation from physiological distribution and background or from the normal tissue activity around the suspect lesion. No respiratory gating was performed.

\section{Results}

F18-FDG PET/CT imaging was negative in 27 patients (39.7\%) and positive in 41 (60.3\%). Widespread metastases involving at least three organs (bone, lungs, lymph nodes etc.) were revealed in 14 patients (34.2\%) (Figure 1, 2), lung metastases in 12 (29.3\%) patients, lymph nodes metastases in 8 (19.5\%), lymph nodes plus lungs in 3 (7.3\%), bone metastases in 3 (7.3\%) (Figure 3), adrenal gland metastases in 1 (2.4\%) patient. In the group of patients classified as G2 according to Fuhrman grading score, F18-FDG PET/CT was negative in 8 patients (67\%) and positive in 4 (33\%); in the group G3 it was negative in 8 (53\%) and positive in 7 (47\%) patients; in the group G4 it was negative in 6 (35\%) and positive in 11 (65\%) patients. In the group of patients affected by clear cell renal cell carcinoma, F18-FDG PET/CT was negative in 17 patients (39\%) and positive in 27 (61\%); in the group of chromophobe cell carcinoma it was negative in all; in the group of papillary cell carcinoma it was negative in 1 patient (34\%) and positive in 2 (66\%) patients; in the patient affected by collecting ducts (Bellini's) carcinoma it was positive; in the group of unknown subtype of renal carcinoma it was negative in 5 patients (31\%) and positive in 11 (69\%) patients. F18-FDG PET/CT results according to Fuhrman score and histological subtype are summarised in Table 1. No significant statistical correlation was observed between F18-FDG PET/CT results and Furhman grade or histology type of primary tumour at contingency tables $(p>0.05)$ despite increasing the Fuhrman grade the percentage of positive studies increases. Sensitivity, specificity, positive predictive value, negative predictive value and accuracy of F18-FDG PET/CT were $82 \%, 100 \%, 100 \%$, $66.7 \%$ and $86.8 \%$, respectively.

In all patients who underwent biopsy of the lesions revealed at $\mathrm{PET} / \mathrm{CT}$, a renal carcinoma metastases were documented. In particular, in 3 patients with bone lesions, F18-FDG PET/CT revealed pathological findings that were not detected by CECT; the diagnosis was confirmed by histological examination after biopsy. Among the 11 patients who underwent a second study after therapy, F18-FDG $\mathrm{PET} / \mathrm{CT}$ showed a disease progression in 4 patients (36\%), partial response in 3 (28\%) and stable diseases in 4 (36\%); no patients showed a complete response. Disease progression was defined as a presence of new lesions and/or higher uptake of the previous metastatic site according to European Organization for Research and Treatment of Cancer (EORTC) criteria [15]; using the same criteria, partial response was defined as a presence of uptake reduction or disappearance of some lesions in absence of new lesions.

\section{Discussion}

Accurate restaging and, particularly, detection of metastatic sites of RC are crucial for the management of patients, because this can lead to modifying therapeutic strategies and serve as an indication for surgery, radiotherapy, or systemic treatment. Despite the fact that the widespread application of US has increased the number of incidentally detected renal tumours, this method has limited utility in identifying retroperitoneal disease, venous tumour 

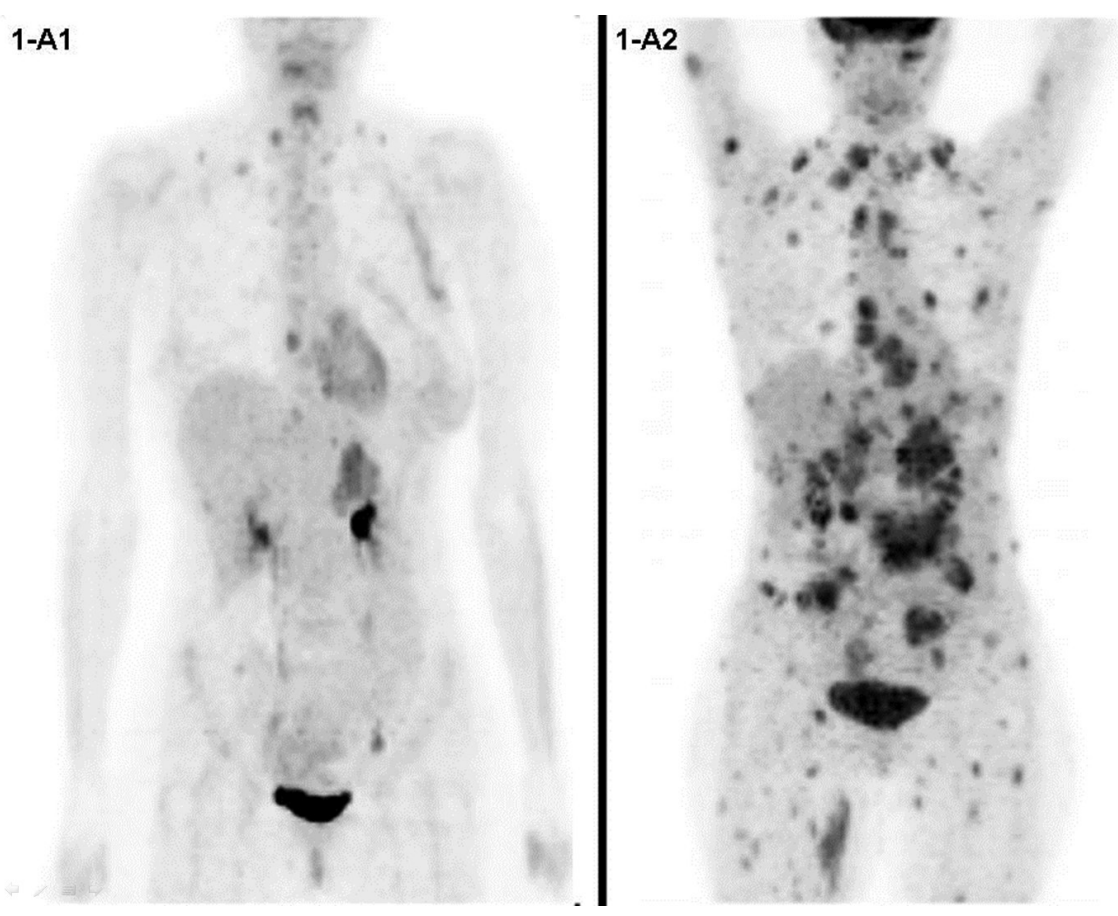

Figure 1. Maximum intensity projection (MIP) images of a patient with widespread metastatic disease who underwent F18-FDG PET/CT before (1-A1) and after (1-A2) systemic therapy. The study revealed a significant disease progression and no response to therapy

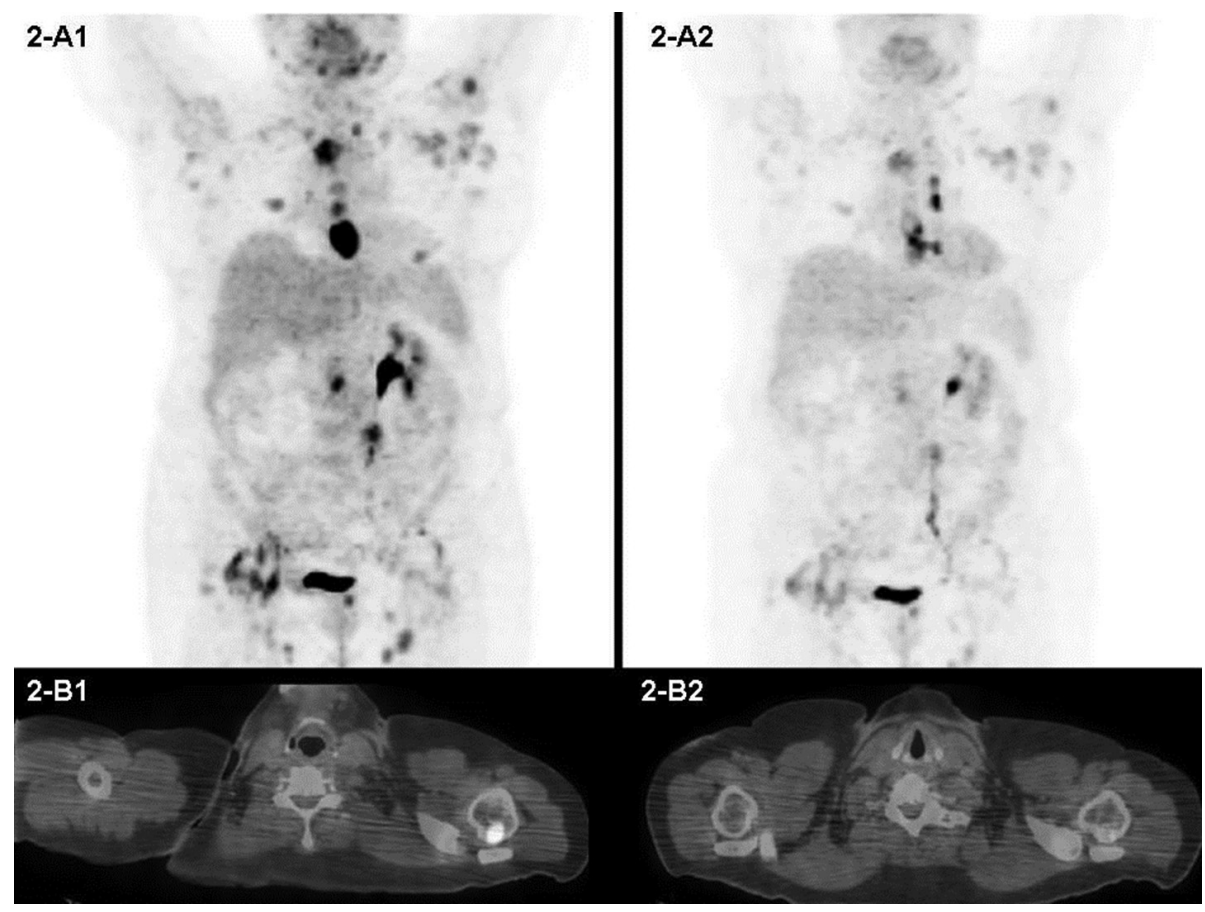

Figure 2. Maximum intensity projection (MIP) images of a patient with widespread metastatic disease who underwent F18-FDG PET/CT before (2-A1) and after (2-A2) systemic therapy with sunitinib. The study revealed uptake reduction in all sites of disease previously detected but showed new lesions at left internal mammary lymph node chain. Axial fused images of the left humerus before (2-B1) and after (2-B2) the treatment revealing partial response

thrombus extension, and metastasis. MRI has some advantages, including multi-planar imaging, high resolution, and lack of radiation exposure, but takes a long time to perform, has limited whole-body imaging capability (necessity to change both patient position and surface coils), and limited accuracy in demonstrating lung metastases. During treatment and follow-up, patients with RC are generally monitored with chest radiograph, CECT and, possibly, MRI to assess the shrinkage or increase of lesions, the burden of 


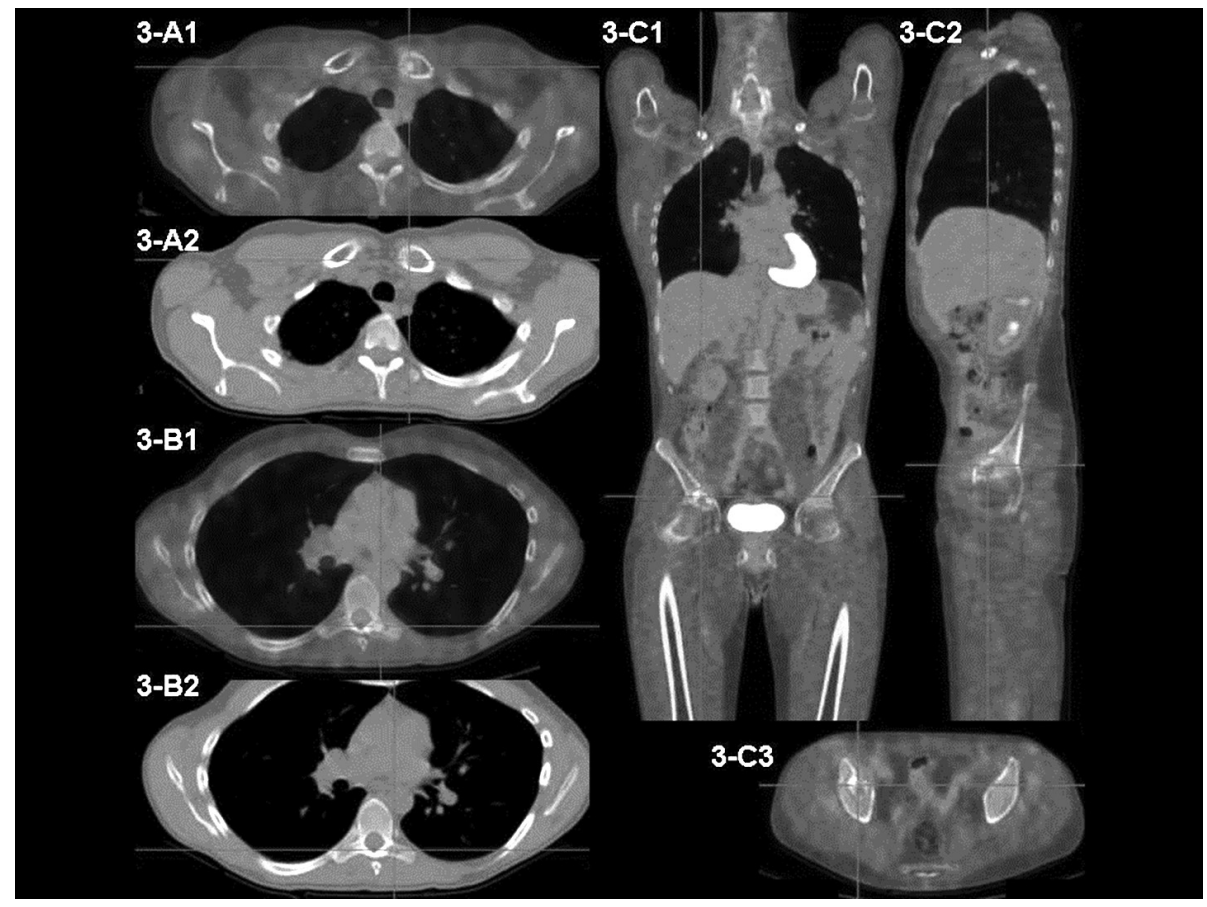

Figure 3. Images of bone metastases in a patient: axial fused (3-A1) and CT (3-A2) of left clavicular lesion, axial fused (3-B1) and CT (3-B2) of dorsal vertebra lesion and coronal fused (3-C1), sagittal fused (3-C2) and axial fused (3-C3) images of acetabular lesion (crosses)

Table 1. PET/CT results according Fuhrman grade and histology of primary tumor

\begin{tabular}{lll}
\hline Fuhrman Grade & PET/CT & PET/CT \\
& positive (\%) & negative (\%) \\
\hline 1 & - & - \\
2 & $4(33)$ & $8(67)$ \\
3 & $7(47)$ & $8(53)$ \\
4 & $11(65)$ & $6(35)$ \\
\hline Histology of primary tumor & PET/CT & PET/CT \\
\hline Clear-cell renal cell carcinoma & positive (\%) & negative (\%) \\
Chromophobe cell carcinoma & - & $17(39)$ \\
Papillary cell carcinoma & $2(61)$ & $4(100)$ \\
Collecting ducts carcinoma & $1(100)$ & $1(34)$ \\
Unknown histological subtype & $11(69)$ & - \\
\hline
\end{tabular}

the disease [16] and the efficacy of treatment, in terms of stable disease, regression or progression, according to the Response Evaluation Criteria in Solid Tumours (RECIST) [17]. CECT represents the mainstay of cross-sectional imaging in $\mathrm{RC}$, with a sensitivity of up to $100 \%$ and specificity exceeding $90 \%$ for retroperitoneal disease, venous tumour thrombus, and metastasis. Recommendations to optimize detection of visceral metastasis of RC include scanning the abdomen and pelvis first, in the arterial phase, followed by scanning of the chest from the lung apices through the liver and kidneys. Although CECT radiation dose increases as a result of thin collimation, over-beaming, and over-ranging, dose modulation software that decreases radiation necessary to obtain a certain contrast-to-noise ratio in the CECT image data when body diameter decreases is commercially available [18].
Since the introduction of F18-FDG PET/CT, a new option and diagnostic opportunity has been available for patients affected by $\mathrm{RC}$. Despite it has been proved to be useful in revealing incidental congenital renal and ureteric anomalies in patients studied for neoplastic reasons $[19,20]$, it is well known and accepted that F18-FDG PET/CT has low sensitivity for the diagnosis of primary RC because of renal excretion of the F18-FDG [21-24]. In fact it has not greatly improved the management of primary renal cancer because the specificity and sensitivity of this technique seem to be less effective than CECT and because primary renal tumours could express low levels of glucose transporters such as GLUT-1, which are responsible for the accumulation of F18-FDG [21]. On the contrary, although there are limited data available, its role for detection of recurrent $\mathrm{RC}$ seems promising and some papers have shown good sensitivity and specificity ranging from 63 to $88 \%$ and 75 to $100 \%$, respectively [21, 22, 25-29].

F18-FDG PET without CT has often demonstrated modest accuracy for metastatic RC, with positive studies being suspicious, while negative studies not reliably excluding disease [26] and being too unreliable to recommend its routine use in the restaging of RC. Most studies have been performed using PET while only few studies, like ours, with PET/CT [22, 25-28, 30-32]. Park et al. [31] have shown that sensitivity, specificity and accuracy of F18-FDG $\mathrm{PET} / \mathrm{CT}$ scanning in detecting recurrent $\mathrm{RC}$ is $89.5 \%, 83.3 \%$ and $85.7 \%$, respectively. Kumar et al have analysed 63 patients who underwent $103 \mathrm{PET} / \mathrm{CT}$ scans with a sensitivity of $90 \%$, specificity of $91 \%$, and accuracy of $90 \%$ in diagnosing recurrent disease [32]. The most important studies and diagnostic performances of PET and PET/CT are summarised in table 2. Our data are in concordance with literature, in particular with those studies performed with PET/CT [31, 32], which is the current technologic standard; it's well established that PET/CT is superior than PET alone giving higher 
Table 2. The most important studies and diagnostic performances of PET and PET/CT

\begin{tabular}{|c|c|c|c|c|c|c|c|c|}
\hline Author & Year & Modality & N.Pts & Se (\%) & $\mathrm{Sp}(\%)$ & Ac $(\%)$ & PPV (\%) & NPV (\%) \\
\hline Safaei et al. [27] & 2002 & PET & 36 & 88 & 75 & - & - & - \\
\hline Jadvar et al. [26] & 2003 & PET & 25 & 71 & 75 & 72 & 94 & 33 \\
\hline Majhail et al. [28] & 2003 & PET & 24 & 64 & 100 & 67 & 100 & 20 \\
\hline Kang et al. [22] & 2004 & PET & 66 & 75 & 100 & - & 98 & 30 \\
\hline Park et al. [31] & 2008 & $\mathrm{PET} / \mathrm{CT}$ & 63 & 89 & 83 & 86 & 77 & 93 \\
\hline Rodryguez et al. [25] & 2010 & PET & 58 & 81 & 86 & 59 & 91 & 73 \\
\hline Kumar et al. [32] & 2010 & $\mathrm{PET} / \mathrm{CT}$ & 63 & 90 & 91 & 90 & 95 & 81 \\
\hline Nakatani et al. [30] & 2011 & PET & 23 & 81 & 71 & 79 & 90 & 56 \\
\hline Present study & 2011 & PET/CT & 68 & 82 & 100 & 86.8 & 100 & 66.7 \\
\hline
\end{tabular}

$\mathrm{N} . \mathrm{Pts}=$ number of patients; $\mathrm{Se}=$ sensibility; $\mathrm{Sp}=$ specificity; $\mathrm{Ac}=$ accuracy; PPV = positive predictive value; NPV = negative predictive value

specificity and reducing false positive results. Compared with a very high specificity and positive predictive value (on average higher than those published in literature), our results suffer from a sub-optimal sensitivity and low negative predictive value. The most common cause for a false-negative result is microscopic disease, in fact it takes a certain number $\left(10^{4}-10^{7}\right)$ of tumour cells with increased abnormal glucose metabolism to be detected by PET/CT imaging. Most of our diagnostic failures of PET occurred in lungs and liver. In fact PET technology even if equipped with CT for anatomic localisation and attenuation correction, could fail to correctly assess small lung metastases smaller than $5 \mathrm{~mm}$; it is unclear whether this drop in sensitivity may be due to pulmonary motion artefacts or to a lower metabolic activity of lung metastases. The use of respiratory-gating and time-of-flight technology (TOF) [33] may improve diagnostic accuracy by reducing motion artefacts, blurring, "smearing effect", and allowing a better spatial resolution; this could ensure a higher accuracy and a more precise SUV calculation, but no dedicated studies have been published on this specific topic. Moreover, our data showed that PET results are not related to Fuhrman score or histological type of primary tumour in concordance with those reported in other studies [28,30].

An interesting field of application of F18-FDG PET/CT seems to be the therapy response evaluation [34]; it has been suggested to assess early response to tumour angiogenesis inhibitor drugs by means of FDG-PET, since both soft tissue and bone metastasis of $\mathrm{RC}$ demonstrate decrease of uptake in therapy responders after one to two months of treatment [34]. Katani et al have prospectively studied 44 patients by three sequential scans performed to determine if the timing of the investigation was relevant (before treatment, after 4 and 16 weeks); they concluded that baseline F18-FDG $\mathrm{PET} / \mathrm{CT}$ yields prognostic significant data and responses occur in the majority of patients after 4 weeks of therapy; however it's not until 16 weeks when the results become prognostically significant [35]. Despite a very small number of patients, in our study therapy response evaluation has been documented as feasible and useful for clinical management.

A critical issue is the histological confirmation of pathologic findings revealed by PET/CT to effectively establish and confirm the presence of relapsed disease, which has to be theoretically considered the gold standard. Unfortunately, in daily practice, this is seldom possible because of clinical reasons, feasibility of the procedure and effective advantages of this approach in absence of a radical surgical intent. In our study histological confirmation was available in 10 patients and all the other ones were compared with clinical plus radiological findings. It's interesting that in 3 cases bone metastases were revealed at F18-FDG PET/CT and confirmed at histological examination in presence of negative CECT. Despite the very small number of cases, this could suggest a possible added value of metabolic imaging, responsible for an increased detection rate of bone metastases frequently present in patients affected by RC. A systematic confrontation between the two methods in a larger series of patients would be desirable in order to confirm or controvert this insight also considering that traditional nuclear medicine diagnostic tools in this field are mainly based on bone scintigraphy with technetium-99m methylene diphosphonate, characterised by limited accuracy. In a prospective study, Sohaib et al. [36] have recently compared MRI and bone scintigraphy diagnostic performances in 47 patients obtaining high levels of specificity in both methods ( $97 \%$ and $94 \%$ respectively) but significantly different sensitivity values (94\% for MRI vs. $62 \%$ for bone scan). Wu et al have compared F18-FDG PET and technetium-99m methylene diphosphonate bone scan documenting higher sensitivity and accuracy of positron imaging in detecting bone metastases in patients affected by RC [37]. The major limits of bone scan seem to be the low sensitivity, especially for small lesions, and that the evaluation is limited to bone.

\section{Conclusions}

In conclusion, despite the limitations of our study due to the retrospective type of analysis and the absence of systematic histological confirmation of pathological uptake, our results are in agreement with literature and suggest that F18-FDG PET/CT is characterised by high specificity and positive predictive value and could be useful in restaging patients with renal carcinoma. This method could be very useful in detecting bone metastases and in therapy monitoring, but cannot be recommended for definitely ruling out suspected disease relapse, due to its low negative predictive value.

\section{References}

1. Ferlay $\mathrm{J}$, Autier $\mathrm{P}$, Boniol $\mathrm{M}$ et al. Estimates of the cancer incidence and mortality in Europe in 2006. Ann Oncol 2007; 18: 581-592.

2. Motzer RJ, Bander NH, Nanus DM. Renal-cell carcinoma. N Engl J Med 1996; 335: 865-875. 
3. Horner MJ, Ries LAG, Krapcho M et al. SEER cancer statistics review, 1975-2006. Bethesda, MD: National Cancer Institute; 2010

4. Rini BI, Rathmell WK, Godley P. Renal cell carcinoma. Curr Opin Oncol 2008; 20: 300-306.

5. Robson CJ, Churchill BM, Anderson W. The results of radical nephrectomy for renal cell carcinoma. J Urol 1969; 101: 297-301.

6. Van Brussel JP, Mickisch GH. Prognostic factors in renal cell and bladder cancer. BJU Int 1999; 83: 902-908.

7. Lam JS, Shvarts O, Leppert JT et al. Renal cell carcinoma 2005: new frontiers in staging, prognostication and targeted molecular therapy. J Urol 2005; 173: 1853-1862.

8. Flanigan RC, Campbell SC, Clark Jl et al. Metastatic renal cell carcinoma. Curr Treat Options Oncol 2003; 4: 385-390.

9. Ahmad T, Eisen T. Kinase inhibition with BAY 43-9006 in renal cell carcinoma. Clin Cancer Res 2004; 10: 6388S-6392S.

10. Rini BI. VEGF-targeted therapy in metastatic renal cell carcinoma. Oncologist 2005; 10: 191-197.

11. Anandappa G, Hollingdale A, Eisen T. Everolimus - a new approach in the treatment of renal cell carcinoma. Cancer Manag Res 2010; 2: 61-70.

12. Mueller-Lisse UG, Mueller-Lisse UL, Meindl T et al. Staging of renal cell carcinoma. Eur Radiol 2007; 17: 2268-2277.

13. Downs TM, Schultzel M, Shi $\mathrm{H}$ et al. Renal cell carcinoma: risk assessment and prognostic factors for newly diagnosed patients. Crit Rev Oncol Hematol 2009; 70: 59-70.

14. Fuhrman SA, Lasky LC, Limas C. Prognostic significance of morphologic parameters in renal cell carcinoma. Am J Surg Pathol 1982; 6: 655-663.

15. Young $H$, Baum R, Cremerius $\cup$ et al. Measurement of clinical and subclinical tumour response using [18F]-fluorodeoxyglucose and positron emission tomography: review and 1999 EORTC recommendations. European Organization for Research and Treatment of Cancer (EORTC) PET Study Group. Eur J Cancer 1999; 35: 1773-1782.

16. Hafez KS, Novick AC, Campbell SC. Patterns of tumor recurrence and guidelines for follow-up after nephron sparing surgery for sporadic renal cell carcinoma. J Urol 1997; 157: 2067-2070.

17. Therasse P, Arbuck SG, Eisenhauer EA et al. New guidelines to evaluate the response to treatment in solid tumors. European Organization for Research and Treatment of Cancer, National Cancer Institute of the United States, National Cancer Institute of Canada. J Natl Cancer Inst 2000; 92: 205-216.

18. Mueller-Lisse UG, Mueller-Lisse UL. Imaging of advanced renal cell carcinoma. World J Urol 2010; 28: 253-261.

19. Bertagna F, Terzi A, Pizzocaro $\mathrm{C}$ et al. Incidental congenital renal and ureteric anomalies in patients studied for neoplastic diseases. Hell J Nucl Med 2010; 13: 177-178.

20. Bertagna F, Terzi A, Bosio G et al. Congenital Triple Kidney in a Patient Evaluated by F-18-FDG-PET/CT for Oncologic Reason. Clin Nucl Med 2011; 36: 937-938.

21. Aide N, Cappele O, Bottet P et al. Efficiency of [(18)F]FDG PET in characterising renal cancer and detecting distant metastases: a comparison with CT. Eur J Nucl Med Mol Imaging 2003; 30: 1236-1245.
22. Kang DE, White RL Jr, Zuger JH et al. Clinical use of fluorodeoxyglucose F 18 positron emission tomography for detection of renal cell carcinoma. J Urol 2004; 171: 1806-1809.

23. Miyakita $\mathrm{H}$, Tokunaga $\mathrm{M}$, Onda $\mathrm{H}$ et al. Significance of 18F-fluorodeoxyglucose positron emission tomography (FDG-PET) for detection of renal cell carcinoma and immunohistochemical glucose transporter 1 (GLUT-1) expression in the cancer. Int J Urol 2002; 9: 15-18.

24. Ozülker T, Ozülker F, Ozbek $E$ et al. A prospective diagnostic accuracy study of F-18 fluorodeoxyglucose-positron emission tomography/computed tomography in the evaluation of indeterminate renal masses. Nucl Med Commun 2011; 32: 265-272.

25. Rodrýguez Martý nez de Llano S, Jime nez-Vicioso A, Mahmood S et al. Clinical impact of (18)F-FDG PET in management of patients with renal cell carcinoma. Rev Esp Med Nucl 2010; 29: 12-19.

26. Jadvar H, Kherbache HM, Pinski JK et al. Diagnostic role of [F-18]-FDG positron emission tomography in restaging renal cell carcinoma. Clin Nephrol 2003; 60: 395-400.

27. Safaei A, Figlin R, Hoh CK et al. The usefulness of F-18 deoxyglucose whole-body positron emission tomography (PET) for re-staging of renal cell cancer. Clin Nephrol 2002; 57: 56-62.

28. Majhail NS, Urbain JL, Albani JM et al. F-18 fluorodeoxyglucose positron emission tomography in the evaluation of distant metastases from renal cell carcinoma. J Clin Oncol 2003; 21: 3995-4000.

29. Ramdave S, Thomas GW, Berlangieri SU et al. Clinical role of F-18 fluorodeoxyglucose positron emission tomography for detection and management of renal cell carcinoma. J Urol 2001; 166: 825-830

30. Nakatani K, Nakamoto Y, Saga T et al. The potential clinical value of FDG-PET for recurrent renal cell carcinoma. Eur J Radiol 2009; 79: 29-35.

31. Park JW, Jo MK, Lee HM. Significance of 18F-fluorodeoxyglucose positron-emission tomography/computed tomography for the postoperative surveillance of advanced renal cell carcinoma. BJU Int 2009; 103: 615-619.

32. Kumar R, Shandal V, Shamim SA et al. Role of FDG PET-CT in recurrent renal cell carcinoma. Nucl Med Commun 2010; 31: 844-850.

33. El Fakhri G, Surti S, Trott CM et al. Improvement in lesion detection with whole-body oncologic time-of-flight PET. J Nucl Med 2011; 52: 347-353.

34. Lyrdal D, Boijsen M, Suurküla $M$ et al. Evaluation of sorafenib treatment in metastatic renal cell carcinoma with 2-Xuoro-2-deoxyglucose positron emission tomography and computed tomography. Nucl Med Commun 2009; 30: 519-524.

35. Kayani I, Avril NE, Bomanji J et al. Sequential FDG-PET/CT as a biomarker of response to sunitinib in metastatic clear cell renal cancer. Clin Cancer Res 2011; 17: 6021-6028.

36. Sohaib SA, Cook G, Allen SD et al. Comparison of whole-body MRI and bone scintigraphy in the detection of bone metastases in renal cancer. $\mathrm{Br}$ J Radiol 2009; 82: 632-639.

37. Wu HC, Yen RF, Shen YY et al. Comparing whole body 18F-2-deoxyglucose positron emission tomography and technetium-99m methylene diphosphate bone scan to detect bone metastases in patients with renal cell carcinomas - a preliminary report. J Cancer Res Clin Oncol 2002; 128: 503-506. 